\title{
NIHILISMO EXISTENCIAL ORGANIZACIONAL FRENTE A LAS UTOPÍAS AMBIENTALES (NEGACIÓN AL VALOR DEL VISIONAR)
}

\author{
Luis Eduardo Quintero Bocanegra ${ }^{1}$
}

\begin{abstract}
RESUMEN
El presente documento invita a reflexionar sobre el nihilismo organizacional, entorno a los sistemas de gestión medio ambiental, el cual hace parte de una derivación de la noción sobre la realidad como resultado de una desilusión y posterior resignación en la ignorancia de los fenómenos, por lo que coarta los planes a futuro y las actitudes de los actores. Obviamente, el ser humano no puede vivir sin una motivación para sus acciones, lo que conlleva de esta manera a colocar los intereses por encima del propósito de los elementos existentes, haciendo que lo importante para los sujetos no trascienda más allá del presente, destacándose el hedonismo y la rentabilidad en el ámbito comercial.
\end{abstract}

PALABRAS CLAVE

Cambio, Gestión, Participación, Organizaciones, Visión.

\begin{abstract}
This paper encourages reflection on organizational nihilism in relation to environmental management systems, which is part of a perception of the reality resulting from a loss of hope and acceptance of the ignorance of reality. This limits future plans and people's motivation. But, the human being cannot live without motivation, resulting in people putting their own self-interest ahead of the existing reality. It allows individual's priorities to stay in the present, ending up with hedonism and profitability in the commercial field.
\end{abstract}

KEYWORDS

Change, Management, Participation, Organizations, Vision.

Fecha de recepción: 30 de junio de 2015.

Fecha de evaluación: 1 de agosto de 2015.

Fecha de aceptación: 8 de septiembre de 2015.

1 Estudiante del Programa de Administración de Empresas de la Universidad Nacional. E-mail: lequinterob@unal.edu.co. 


\section{INTRODUCCIÓN}

Existen diversos sistemas de gestión medio ambiental (SGMA), entre ellos, unos con gran aceptación mundial debido al respaldo institucional de carácter oficial, lo que los faculta expedir certificados de cumplimiento a partir de requisitos mínimos, estos sistemas no son prescriptivos, en su mayoría, y posibilitan ser adaptados; sin embargo, depende de la jurisprudencia local, en SGMA podemos destacar las normas ISO 14000 y la EMAS de origen europeo, que son parte del mundo empresarial y no han trascendido de manera reconocida al público en general, conllevando que en Latinoamérica no se les perciba como un método para un cambio de época. (L. Perea, 2011)

La cuestión es ¿cómo la organización considera los SGMA y la naturaleza de la política de implementación?, es cierto que estos sistemas son para la disminución de los impactos ambientales; sin embargo, los intereses tras su concepción influencian su existencia, por ejemplo, la superposición de fines sobre medios junto a consideraciones del talante: “...esta etiqueta contribuye con nuestro good will", "reduce nuestros costos implícitos", "expía nuestras culpas y podemos trabajar más tranquilos", "apliquemos este sistema, pues es la megatendencia del mercado", "los mencionan como alternativas innovadoras", entre otras. Estas nociones guiadas por intereses ajenos a la esencia del SGMA afectan las ejecuciones en el largo plazo, por eso es de aclarar que aquellos "supuestos ocultos" determinan a la empresa desde la casuística de las operaciones internas, donde los actores participan como autómatas de la directriz, ignorantes, fungiendo como herramienta de las políticas de gestión que son influenciadas por los diseñadores, mas no por la naturaleza del sistema. Por tanto, la clave va más allá de la reducción de impactos ambientales, es buscar el bienestar integral en comunidad, implicando participación total de las partes en la empresa comercial, sitio donde se ridiculizan o enaltecen las decisiones en cuanto a dichas gestiones; por lo tanto, es necesario reconocer la mentalidad y funcionalidad del sistema en el cual se enmarca el conglomerado organizacional, para convertirse en gestor de cambios posibles mediante el abandono a la resignación a lo establecido por la realidad.

\section{Caracterizaciones}

A nivel territorial Colombia está ubicada unos kilómetros por encima de la línea del ecuador, en zona horaria UTC-05:00, cruzada por la cordillera de los Andes de noroccidente a sur, además, es un país occidental del trópico, con doble salida al mar, con territorio en la selva del Amazonas, lo que confiere potencial para incursiones científicas por la diversidad existente, adicionalmente tiene condiciones aptas para la implementación de energías alternativas y el ecoturismo.

El gobierno colombiano a mediados de la segunda década del siglo XXI afronta un proceso de paz después de aproximadamente 50 años de conflicto interno contra una guerrilla que argumenta un ideal desde las armas, además se presenta un reconocimiento del concepto de competencia como política de desarrollo desde el Plan Nacional 2015, del cual existen rumores de insolvencia presupuestal, se observa también un auge en la participación de los ciudadanos del común en diversos productos financieros, de los cuales se destaca el crédito. Por último, cabe recalcar la tendencia del pago electrónico avalado por la cultura del microchip, respecto de lo anterior la situación genera un ambiente comercial propicio para la inestabilidad donde el estado de bienestar social deberá intervenir constantemente en contravía de los desmanes del gendarme, en este caso la forma de actuar es "tanto mercado como sea posible y tanto estado como se requiera”, llamada por el gobierno de turno como Prosperidad Social (palabras presidente Juan Manuel Santos, 2011) con origen en la llamada Tercera Vía, dicha concepción de gobierno para las empresas colombianas muestra un modelo de desarrollo traducido en garantías de libre competencia y estabilidad en el mercado interno, pero, sobretodo, una promesa de rentabilidad basada en confianza al ideal capitalista como motor de desarrollo de la sociedad. Es de anotar que el Estado pretende contener los focos de inestabilidad propiciando el desarrollo a través de la competitividad de diferentes escenarios cotidianos. 
A nivel social Colombia es un país voluble y subdividido, tanto ideológica como culturalmente, pero en su mayoría es de carácter hedonista y cooperativo, y aún más, en situaciones de urgencia sus individuos son creativos, emprendedores, preocupados, empíricos, impulsivos, y gestores de sus emociones, dándose los insumos de donde afloran innovaciones, es decir, en ultimas existe diversidad de capital humano como para tener tantas opiniones y alternativas de solución que sobrepasan las capacidades de acople y apoyo; aun así es un país desaprovechado, las personas con iniciativa casi siempre están sin músculo financiero, y los que logran conseguir un soporte fiscal se ven dependientes de empresarios, claro está es implícito esa consecuencia de la aventura financiera, pero por qué atar las investigaciones y proyectos ambientales, exclusivamente, a dinero y rentabilidad en contravía al bienestar integral que generaría a largo plazo para los implicados.

Entrando en materia, si el nihilismo es comprendido como la corriente del estudio de la nada, donde los principios de sentido existencial no valen lo suficiente como para interiorizarlos y sentir que es lo único que puede llegar a ser verdad, tanto es así que no vale la pena exigirse en diferentes situaciones. Mejor divagar conforme a la realidad existente, es más puede tener razón pero el interés se desvanece conforme el nivel de agrado; esta corriente ha sido tratada desde el siglo XIX con muchas variaciones por lo que sus interpretaciones dependen del contexto y propósito.

Esta mezcla de organizaciones, sociedad, y la situación nacional, convergen en una lucha constante donde las desilusiones e impotencias de la mayoría de individuos llevan a menos las visiones de un país más civilizado, obligándoseles a adaptarse a la realidad sin pensar cambiarla y aceptándola con resignación, esta posición pasiva frente a la realidad cala profundamente en el subconsciente de las personas que ya se comportan de cierto modo y meditan menos el futuro de manera crítica, ya no les vale visionar debido a la inutilidad práctica del ejercicio, entonces, les resta seguir el cauce de la realidad y aplicar lo ya hecho, pensar lo ya resuelto y sentir la tendencia, aquella pasividad es un indica- dor de una sociedad estancada, adicta al entretenimiento y la satisfacción personal, conviene preguntar el porqué de las cosas y cuestionar el cómo, si la pasividad en sentido progresista se apodera de los núcleos sociales más grandes. Pronto volveremos a las guerras para conseguir lo que nos urge y esperar milagros para lo que no entendemos, debido al estancamiento de dichos núcleos sociales.

Ahora los que se cuestionan y buscan ser agentes del cambio se concentran en centros de investigación o se aíslan en el entorno académico dejando la praxis para los que la necesitan para vivir, les es más fácil cambiar las bases del conocimiento que hábitos arraigados en la cultura general de un país, y habrá que dejar la tarea del cambio a los que se acostumbraron a difundir información, estas personas curiosas a partir de comprender el funcionamiento se predisponen para imaginar nuevas maneras, generando bases conceptuales, pero no es suficiente si no es complementado con visiones a largo plazo, pero estos anhelos que se persiguen con acciones dinámicas (emprendimiento) dependen mucho de los detalles, debe advertirse que estos destellos de oráculo mal implementados provocan también ocasos de las nuevas maneras, por lo que se hace difícil visionar y ejecutar con la costumbre de los malos procederes y desorientación.

Alejados los agentes del cambio del engorroso procedimiento, quedan ideas sueltas y percepciones incompletas, resultando en un conglomerado social amante de la técnica con rumbo dictado por el sistema económico reinante, donde todo tiene un tiempo de existencia limitado y el sentido/razón de las cosas se resume en el placer que puede proporcionar, tanto así que el sentido por juicio combina perfectamente con el sentido comercial, instaurando sus bases en las necesidades de tercer nivel de Maslow (filiación) permeando como necesidad adquirida quedándose así hasta que cambie de nuevo la mentalidad de la comunidad, en términos coloquiales "una concepción tierna de la existencia se arraiga en la mente y es mantenida por el pan y circo a través del tiempo", por lo que una intuición viciada determina ir con la corriente y como a este movimiento le sigue, en consecuencia, la identidad que genera el conglome- 
rado social, amarra la capacidad de juicio de los individuos, siendo ellos los mismo tercos promotores de los ideales de la masa, obligando a salir a flote un instinto de manada aun en el postmodernismo... Resumiendo en sentido curioso: ahora está de moda ser fan.

Si ya no es válido ser un visionario se debe ser reductor de la técnica, seguidor de modas, repetir retahílas enseñadas por el mercado, aglomerar datos, sobrevivir, depender, copiar...; dicha posición pasiva permea la realidad organizacional y, sobre todo, si su propósito es acomodado empezará a tomar fuerza con el tiempo tornando a una realidad endémica que poco a poco acaba con el ente social, dicha pasividad termina convirtiéndose en cegadora de visionarios y coercitiva de la sociedad crítica; en consecuencia, aquella venda de actitudes provocará utopías dejadas a los jóvenes y a los locos de antaño, externos a la organización, mientras dichas estructuras en respuesta a los bichos raros realizan actividades esporádicas que son más que suficiente para contribuir a una causa extraña y sin sentido práctico, es una de las situaciones de los SGMA en las organizaciones guiadas por el dinero y las tendencias del mercado.

A pesar del dinero y el afán que provoca acumularlo, en este modelo capitalista se requiere para el futuro un sistema de transacciones sostenible en volumen y calidad e impacto ambiental, extendiendo más que la vida de los productos la capacidad del planeta y el rendimiento de los recursos limitados, como menciona en conferencia en la Universidad Nacional, “...un modelo infinito no puede coexistir con unos recursos limitados" (Aktouf, 2015), lo poco ilimitado concerniente a los objetos es el servicio, casualmente es en una de las cosas en las que se destaca la gente colombiana, aun así esto no resolvería el problema que nos concierne, dicho de otra manera, aunque se posean opciones, estas no se pueden considerar como solución milagrosa, se necesita ser más profundo en el análisis y lúcido para comunicar la mezcla óptima variable, el pilar es ser agentes del buen cambio y tomar posición participativa en la modificación del entorno que, en principio, es una de las potencialidades que puede ofrecer la empresa a la sociedad.
Es curioso hablar sobre el nihilismo cuando una organización tiene un propósito y le da sentido a sus procesos internos en pro de la consecución del objetivo, se pensará, entonces, la existencia de una incoherencia desde el título, pero es más incoherente hablar sobre intervencionismo en un sistema idealizado en el gendarme; en realidad los SGMA no están siendo aplicados o interiorizados por la mayoría de las organizaciones y solo se implementan porque algún neófito los mencionó o una autoridad circunstancialmente de mayor rango lo impuso; es así como se satirizan los medios, ignorando la verdadera esencia, no se propone crear un SGMA a medida para ser más competitivo mediante el derecho a una etiqueta o por rigidez legislativa, pues en ultimas se es razonable para el ecosistema circundante e indirectamente positivo en relación al consumidor y la ley; por lo tanto, el sentir de la implementación no debe ser influenciado por ideales efímeros, de ser así naturalmente al ocaso de la época los fines serán inherentemente un chiste y la posteridad tendrá el derecho de juzgar los actos de su ascendencia, tanto así es la magnitud de la falencia de conciencia, que deseamos cuidar el medio ambiente por el anhelo del mercado y la asunción pasiva de medidas preventivas y de contingencia, en si la ideología del nada reside en la organización desde la dirección y, por tanto, en los insumos de información que esta posee, es más la empresa está tan cómoda en estructura que solo los avances tecnológicos de otros lugares la despierta y la pone a pensar y cambiar mientras comprende el cómo de lo nuevo, continuando de esta manera desvirtuándose en el tiempo como agente de cambio y precursora de revoluciones.

Es de carácter redundante recordar que son las organizaciones como conglomerado. La gestora principal de los cambios mundiales, después de la naturaleza y el pueblo, es poderosa, y se ha olvidado que supera a los gobiernos representativos; si no se toma en cuenta su esencia y poder popular, por tanto el conglomerado de organizaciones es capaz de modificar la historia y es la amenaza latente de todo gobierno, tanto que hasta los monopolios, o en caso extremo los oligopolios son legítimamente controlados. $\mathrm{Si}$ a las organizaciones se les niega el poder concentrado es porque demostraron que son 
insanos sus actos intencionados, en consecuencia, la mayor fuerza autónoma de cambio junto con otras menores le negaron dicha facultad de poder, y no es lógico para la rentabilidad organizacional ir en contravía; entonces, a la organización comercial le queda seguir el cauce de la realidad, aplicar lo ya hecho, pensar lo ya resuelto, y sentir la tendencia, unos nihilista frente a la participación en el cambio, pues es más fácil disfrutar de las oportunidades del mercado y sus tendencias, que ir con sacrificios propios a buscar la continuación de nuestra civilización.

\section{Pasos constantes}

Propiciar cambios bruscos daña a la organización, en la mayoría de los casos le resta parte de su esencia, llevándola a pique, pero si se desea el cambio se tendrá que precisar la estructura acorde al horizonte planteado con acciones en ritmo adecuado para la tarea, para ello es necesario crear independencia del tradicionalismo adquiriendo hábitos distintos, por ejemplo, la dependencia energética por combustión para el desarrollo de las naciones o la información contable sin análisis de juicio, en general; por lo tanto, cambios progresivos y cuidadosamente manejados procuraran éxito en la consecución de un buen camino hacia las utopías ambientales, la cuestión está en las mutaciones de identidad de otros entes involucrados por sinergia sistémica; aclárese que esta no es una posición adicta al cambio, por el contrario, es dependiente hacia la realización de la visión ambiental, por lo que todos los cambios no son óptimos y menos en todo momento.

Planteado que no es una adicción al cambio, sino una necesidad para la vida empresarial, la posición tendrá que cambiar a ser participativa en modificar el entorno, pasando de ser una fuerza indirecta a una directa, la razón es simple: si logra ser el controlador o fuerza determinante del rumbo del futuro, las debilidades y amenazas no afectarán con el mismo impacto, en cuanto a los SGMA si se logra controlar los efectos adversos producto de un consumismo agresivo, la estructura de costos no se verá afectada de igual manera a empresas comunes. En cuestión de lustros se notará el cambio por la resistencia diferenciada de aquellas estructuras, por tanto, una de las ventajas que generan los buenos SGMA es que son herramientas potenciales en la modificación de la relación hombre/ naturaleza en función del bienestar integral de las criaturas. Los sistemas aquí comprendidos, básicamente, son parte de la planeación de las empresas pero enfocados no solo a actividades internas, sino a actividades de relación que implican la inclusión del entorno y una actitud proactiva complementaria a la preventiva y contingente; en consecuencia, se incita a encaminar el cambio en los demás ámbitos como comerciales o políticos a partir de la presión de un conglomerado organizacional coordinado, tal unión se necesita para acelerar el cambio de manera que se produzca una independencia energética del petróleo como mínimo, y de otro tipo de organizaciones con productos necesarios pero nocivos para el ambiente, igualmente, generaría un auge de la curva de aprendizaje en implementaciones diversas a diferencia del hoy con costes altos en SGMA, debido a la demanda/oferta de entes súperespecializados que trabajan casi aislados, estas son algunas pocas razones del porqué de un sistema participativo.

Continuando, dentro de la organización se establecen insumos para la toma de decisiones y desarrollo de la actividad social, uno de los principales es el que proporciona la contabilidad empresarial, por lo tanto, de ella se requiere un compromiso especial como aporte a los sistemas de gestión más allá del teórico y procedimental, ahora se solicita una contabilidad crítica e irremplazable por ordenador, además que la información proporcionada contenga realidad histórica y conciencia autónoma logrando convertirse en influencia del cambio futuro, siendo deseable unas personas preparadas para aceptar o hacerle frente a ideologías administrativas ambientales, aunque el área como tal no tiene el poder para tomar las decisiones finales si es un pilar de acciones, y como personas poseen conciencia para evaluar actos y principios aplicados, la contabilidad en esencia es una herramienta de control y para tal tarea se le necesita desde dos perspectivas: racionalidad controladora y perspectiva social (F. Quinche, 2008), respectivamente, una para efectos en el corto plazo y otra para implicación decisoria, reiterando que además la contabilidad media las 
relaciones sociales a través de la legitimación de un modelo, teniendo la capacidad de transformar entornos específicos desde la naturaleza representativa de la realidad (Sunder, 2005).

Se influye en la toma de decisiones de la dirección según las circunstancias en la que se presenta una información o se recalca de manera extraoficial la importancia de algún dato; es más, existe una situación reciente donde la teoría contable se presenta como obstáculo al registro ambiental frente a los SGMA mostrándose como anunciante de riesgos, siendo así paranoica y no una herramienta de trato, negándose así como participante y posicionándose como escudo a los sistemas (Larrinaga González,1999), es importante revisar detenidamente cómo la contabilidad a partir de sus bases teóricas puede cambiar a la organización en conjunto con la determinación y análisis que surjan de la relación administración-contabilidad, permitiendo aportarle al ámbito contable una visión sobre las implicación de la información en sistemas de gestión, en este caso en sistemas medio ambientales, dicha perspectiva puede enriquecer las bases teóricas contable aplicadas en la empresa creando un modelo adaptable de costos en el manejo de recursos en función del SGMA, la idea anterior, varias veces ha sido mencionada y faculta a los estudiosos del tema a exigir dicha postura, sin embargo, se debe considerar en este método la ejecución pensada sobre la duda cientifica y construirlo entorno a las visiones utópica de la organización, a manera de ejemplo una organización que implemente un SGMA para tratar el impacto sobre la cantidad de emisiones de carbono de su actividad comercial con una meta periódica más no utópica en la planeación del sistema, pronto se dejará alcanzar por las demás empresas pares en el mercado y la diferenciación inicial será inexistente, por lo cual adjuntarle una visión mayor al SGMA permitirá avanzar conforme la ambición y capacidad de la organización de manera activa y autónoma.

En cuestión de detalles la omisión no deliberada se convierte en niebla de los sistemas que en ultimas le compete a la administración, esta atención es propia del corto plazo y con su debida importancia se debe velar, pues es bien sabi- do: la suma de decisiones determina el curso de la situación final, debido a que con detalles es donde juega la directriz general de la alta gerencia la instauración del sentir original del SGMA, obviamente, la administración es considerada como la directora de todo, pero en realidad sus niveles de acción en una organización varían conforme a la proximidad en la definición de la estrategia, como mínimo se deberá garantizar la claridad del direccionamiento de los esfuerzos, dejando claro la visión a largo plazo y lo que se quiere lograr con lo establecido, las formas de lograrlo puede que difieran o estén enmarcadas en lo obligante, pero si se logra concientizar sobre los detalles se tendrá un proceder distinto que determine en el corto plazo y colabore a lo planeado en el largo plazo, considerando que descuidar un sistema tiene consecuencias entrópicas y desgastantes para una organización instaurada bajo el paradigma de la complejidad y la lógica constructivista, por lo cual, considerar los efectos sistémicos resultado de las interacciones resulta útil (P. Collerette, 1998).

Siguiendo la consideración de detalles en el corto plazo, se retoma un aparte del libro La consultoría de empresas de la Oficina Internacional del Trabajo de Ginebra... "el acercamiento ha estado en la investigación de procesos, situada entre el ámbito científico y la práctica" (OIT, 1980), de esta manera se hace alusión a la inclusión de análisis del entorno dentro de los procesos internos que, por presión, deben incluir apartes del entorno que hacen ineficientes las operaciones dentro de la organización. Es una combinación entre cuestionamientos científicos y la practicidad de lo cotidiano en la búsqueda de la eficacia que se ha desarrollado durante siglos, es esta situación la que permea el corto plazo y plantea el camino hacia el largo plazo, por lo que una incursión en la contabilidad empresarial de este tipo de funcionamiento no estaría de más, obviamente las investigaciones tienen que incluir más que situaciones existentes y plantear escenarios posibles en los que la estructura de costos sea más que una buena idea, desde luego, estas investigaciones son optimización de combinaciones existentes y no una incursión científica de invención propiamente dicha debido a que es necesario empezar a aplicar la mayoría de investigaciones olvidadas o pasadas por alto. 
La gestión empresarial comprende diferentes modelos, pero en su mayoría reina la gestión por resultados, la cual se enfoca a parámetros medibles de muchas situaciones, permitiendo de tal forma al interesado tener información progresiva de muchas de las operaciones, dicho modelo de gestión en principio irá en contravía con una visión utópica ambiental al no permitir incluir aspectos cualitativos en su resultados, otros dirán que el resultado de algo puede ser un documento, pero lo entendido estrictamente a la gestión por resultados es medible y, por tanto, considerado objeto, dicho documento se convierte en un resultado como cantidad/producto y no por su contenido, la alusión "en principio " es debido a que las visiones a largo plazo utópicas no son mensurables, pero en el corto plazo muchas de las acciones pueden ser evaluadas, pues la directriz general conforma un horizonte, avanzar es resultado de los procesos y decisiones reales que tienen un método apropiado de identificación de progreso, por consiguiente una negación total a visiones utópicas en los SGMA por poseer un modelo de gestión empresarial por resultados no es una respuesta concreta, aun así en caso extremo de fundamentalismo en lo medible se argumenta la creación de un modelo ideal de empresa (tipo supuesto económicos múltiple valores) constituyendo una evaluación cualitativa a proyectos a base de comparación por aspectos, ofreciendo un porcentaje de progreso que en últimas se convertiría en una cifra referente.

Un modelo de comparación frente a una situación ideal (Kepner \& Tregore, 1989) se ha abordado durante años para la resolución de problemas desde una análisis de toma de decisiones, para este caso a manera demostrativa y con algunas variantes, se sugiere para dar luz al método constructivo; siendo un modelo, una abstracción simplificada de la realidad para entenderla, en este se destaca información en la toma de decisiones a partir de la comparación de escenarios con una estructura macro de: primero, evaluación; segundo, análisis; y tercero, diagnóstico. Para tal búsqueda de avance en pro del horizonte "utópico" se requiere un estudio por escenarios reales y posibles, por lo cual se plantean cinco escenarios: visión, bien después (proyectos futuros), ya casi (proyectos en ejecución sin terminar), ahora, y el nada.
La razón de ser de los cinco escenario es simple, se requiere crear una evaluación sobre la noción de avance, este lleva a evaluar los hipotéticos de las acciones a realizar desde el ahora, debido a que es un modelo de comparación frente a la visión utópica, se realiza de a pares: visión vs ahora, visón vs bien después, visión vs ya casi, ahora vs nada; el primero es para un control de horizonte, el segundo para el hipotético avance con proyectos futuros representado la potencialidad de avance, el tercero es para saber la posición final al culminar los proyectos en ejecución, y el cuarto es para saber el valor real en aspectos de lo que es existir para la empresa. De modo que se busca comprender cada escenario desde su comparación, construyendo una lista de aspectos comunes que sean un estándar para la evaluación, se determinan niveles de relevancia de los aspectos de manera ascendente: $\mathrm{X}$ nada, 1 deseable, 2 importante, 3 necesario; es de anotar que las comparaciones se hacen relacionado lo utópico con lo real por lo que es frente a algo perfecto, sin embargo, queda a la decisión del interesado la relevancia de los aspectos, y según la medida de relevancia se optarán por porcentajes, comprendiendo que lo utópico es el 100\%; para las comparaciones dos y tres (bien después, y ya casi) se pueden hacer separando por proyectos o de manera conjunta, lo importante es comprender que existirán proyectos que siempre sacarán del camino hacia lo utópico aunque sean muy beneficiosas en el corto plazo, la decisión siempre quedará en manos del interesado.

Lo anterior ha sido solo la fase de evaluación, pero no la más importante porque en el análisis y el diagnóstico es lo que realmente interesa al ente decisor, a nivel de análisis se recurre desde lo técnico y casuístico del escenario, por lo que ayudas con los dofas aumentados suelen ser de gran aporte pero no las únicas herramientas, pues existen otras como six sigma. En el diagnóstico se recurre a la inteligencia del interesado para mostrar las situaciones posibles donde la variabilidad relacionada con la probabilidad y el impacto son determinantes; es de aclarar que un modelo que combina una evaluación subjetiva y unos análisis de escenarios posibles no amarra a los interesados a realizarla de una manera determinada, además, trabajar sobre 
Figura 1

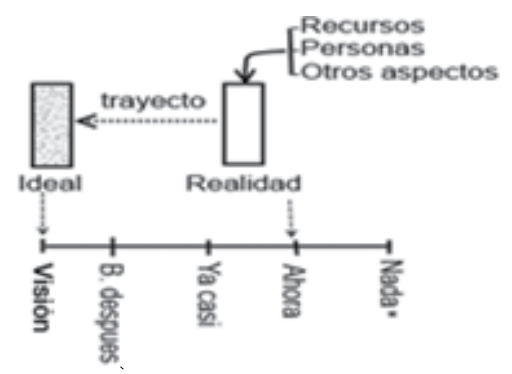

\begin{tabular}{|c|c|c|c|c|c|}
\hline Aspecto & Visión & B.D & Compración & Y.C. & Comparación \\
\hline A & $\cdots$ & - & $33 \%$ & $\cdots$ & $66 \%$ \\
\hline B & $\cdots$ & - & $33 \%$ & $\times$ & $0 \%$ \\
\hline C & $\cdots$ & - & $33 \%$ & - & $33 \%$ \\
\hline D & $\times$ & - & + & - & + \\
\hline$\infty$ & $\cdots$ & $\cdots$ & $100 \%$ & $\cdots$ & $100 \%$ \\
\hline & $\cdots$ & $\bar{X}$ & $55.33 \%+$ & $\bar{X}$ & $55.33 \%+$ \\
\cline { 5 - 7 }
\end{tabular}

\begin{tabular}{|c|c|c|c|}
\hline Aspecto & Futuro & Ahora & Comparacior \\
\hline A & $\cdots$ & $\times$ & $0 \%$ \\
\hline B & $\cdots$ & $*$ & $33 \%$ \\
\hline C & $\cdots$ & $*$ & $33 \%$ \\
\hline D & $\times$ & $\times$ & $\times$ \\
\hline$\infty$ & $\ldots$ & $\times$ & $0 \%$ \\
\hline
\end{tabular}

En algunas circunstancias la evaluación es definitiva para la toma de decisiones, pero aquí solo es un indicador de progreso o de recesib́n, por lo que es altamente subjetiva, sin embargo, crea una nocid́n de propdsito en las decislones futurass lo gue realmente lmporta es el andlisis $y$ su diegndentico por decislones futuras; 10 que realmente importa es ef ansisis y su diagndstico por escenarios, pues puede darse la situación en el que ahora está más cerca de la visión que los proplos la situación de provectos futuros, o que los provectos en corto plazo son beneficiosos pero alejan del ideal; en este caso los provectos futuros y por finalizar dejan en el mismo porcentaje de avance demostrando que es altamente engahioso tomar el dato grueso como pilar de la decisión, es de recordar que un indicador no demuestra el detalle de la realidad por lo que en el antlisls se debe conternplar todos los aspectos tomados, creando una ldea de anbilisis se debe contemplar todos los aspectos tomados, creando una idea de lo ocurrida, para este modelo es de esperar que la evaluación sea menos importante que el analisis y diagndstico respectivos.

-En este grafico no estíb plasmado y se deja a auto consideración

Fuente: Elaboración propia del autor.

números para encontrar objetividades se vuelve algo confuso dentro del imaginativo de lo utópico por lo que es mejor trabajar con escalas absolutas de realidad, ocurre o no y su factibilidad. Al final cada quien dependiendo de sus requerimientos personalizará el modelo y lo adaptará para su uso, de hecho aquí se muestra de manera tan general y sin mucha complicación para incentivar añadidos.

Otro aporte importante es el aspecto ingenieril en la traducción de efectos ambientales a costos medibles y consignables para hacerlos datos trabajables, dicho entendimiento es crucial para que muchos proyectos en el corto plazo sean juzgados, sin embargo, no es el único aporte a considerar, en la ingeniería reside implementar un sistema útil para la empresa, sobre todo capaz de armonizar con el objetivo empresarial siendo controlable y práctico, por ejemplo, la disminución de costes en diversos insumos y servicios usuales de toda empresa como lo son el agua, la electricidad, el papel, fletes, comida, entre otros, que si llegasen a ser instaurados aisladamente perderán utilidad y provocarán un cuello de botella para la organización mediante confusiones, dicha área técnica es importante para construir paso a paso el camino hacia el horizonte, debe estar en constante charla con el área contable, administrativa, y con los responsables del funcionamiento de los sistemas de información, por lo que una mezcla óptima de las anteriores depende de la capacidad y ambición de la empresa, sobre todo de que tanta inteligencia se involucre en el diseño, planteamiento y ejecución del sistema de gestión ambiental.

\section{CLARIFICACIONES DE PARTICIPACIÓN}

Un SGMA sin visión a largo plazo es común en la ignorancia del entorno sistémico, pero es extraño uno que contenga una visión de cambio participativo; retomando una clarificación de la situación es de notar que existe en la historia colombiana un continuo listado de fracasos en obras civiles y descontento en la mayoría de incursiones técnicas, en el país los proyectos fracasan por tiempo o por ahogo del presupuesto, los ingenieros son los entendidos en los temas de camisas de fuerza para muchos de sus planes, aun así aprenden rápido y se acoplan sacando adelante exitosos proyectos que son reconocidos, incluso a nivel internacional, sin embargo, los ingenieros y la sociedad olvidan muy rápido los fracasos pasados de sus actuales héroes, permitiendo de esta manera que aún se quiera y se confié en los proyectos ingenieriles, aun así mu- 
chos de los proyectos no entienden la naturaleza de la generación que los solicita y la generación futura que les dará uso, por lo que muchas de las incursiones al ser fundadas tiempo después rápidamente se vuelven obsoletas, trayendo costos inflados a los que financian tal obra y engorrosos procedimientos de adecuación.

En caso tal que una obra logre estar en condiciones de capacidad de uso necesariamente tiene que tener procedimientos de mantenimiento, y este país se caracteriza por el "mantenimiento constructivo", es una manera de caracterizar que nuestras reparaciones no son hechas a medida sino, hechas conforme los materiales existentes y elementos a la mano, por lo que es normal encontrar remiendos que destacan visualmente y que en principio donde sean dispares reducen el rendimiento de los objetos, no está mal querer conseguir la prolongación de la vida útil, pero si continuamos con ese pensamiento de no mantener a tono todo, seguirá la costumbre de permitir fallos, obviamente en la cotidianidad luchamos contra la obsolescencia programada de los productos, pero si se realiza un proyecto y es construido desde cero, ¿por qué tienen que luchar las mismas organizaciones clavándose la inutilidad programada de sus acciones?

La visión utópica de la que debe nutrirse el sentido de un SGMA debe luchar contra la predisposición de los grandes proyectos que saben cuándo se empiezan pero no cuándo se terminan desde la posición participativa, se marca una actitud de acción permitiendo dar pasos hacia el horizonte propuesto, aun así tiene otro propósito y es trascender en las generaciones mediante la comprensión de las necesidades y como las abordarán las futuras, si les será útil, práctico, objetivo, eficaz, entre otras cualidades que marcarán más que un proyecto, un modelo de existencia demarcado por la actitud organizacional propuesta, por lo que se requiere una contribución contable de control, realidad social y disposición de información de manera especial en conjunto con una administración de corto plazo enfocada en los detalles, además una alta gerencia estratégica liberadora de la energía del sistema, y unos proyectos de ingeniería que preocupen en conjunto, en presupuesto, en tiempo $\mathrm{y}$ en impacto social, diseñados para tener un adecuado mantenimiento que prologue la vida de estos, y si han de implementarse deben estar en armonía con el objetivo empresarial y en constante interacción con otros SGMA internos o externos, de manera tal que el impacto sea fuerte y preparado para la organización en la lógica empresarial, buscando la reiniciación de los conglomerados organizacionales en pro del cambio mediante la independización de sus estructuras de costos de las tendencias del mercado y aplicarles un proceder de funcionamiento que trascienda en el siglo. Obviamente en proyectos que buscan trascender y ser utópicos al inicio poseen riesgos y variaciones, por lo que se requiere especial atención en la determinación, diseño, planeación y ejecución inicial, es aquí donde los detalles deben estar cuidadosamente considerados, junto a otras ramas antes expuestas.

Figura 2. Funcionamiento global al rededor del SGMA utópico.

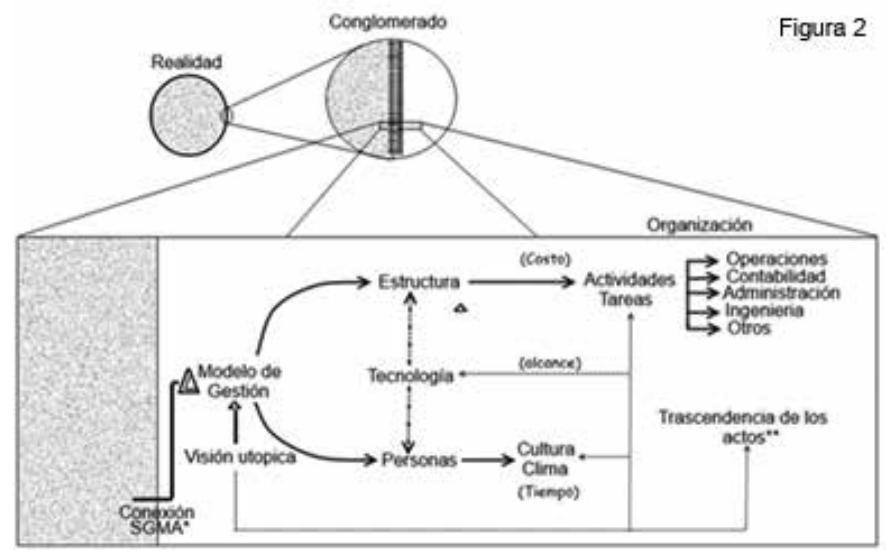

Fuente: Elaboración propia del autor. 
En el ente se requiere por tanto un manejo de tiempos, costos y alcances para ir construyendo tras el horizonte utópico planteado, evitando reiterar en inversiones para adecuar proyectos que se constituyeron como soluciones a otros "elefantes blancos" ineficientes, que ahora están fuera de capacidad; se requiere, pues, una peculiar orientación en el modelo de gestión que se nutre por la estructura y las personas involucradas, donde la tecnología es un puente de interacción entre la realidad organizacional y el mundo particular de las personas, dicha estructura dispone de actividades, tareas u operaciones que se agrupan en procesos gestionables y son influidos por áreas distintas, ya sea ingeniería, manufactura, mantenimiento, finanzas, contabilidad, administración, etc., en cambio en el área personal se observan unos ambientes propiciados por la cultura, un clima, y dimensiones de personalidades aisladas, comprendiendo todo lo anterior esto se puede plasmar un bosquejo a modo de resumen y abstraer la idea de cómo la parte interactúa con la visión utópica y la posibilidad teórica de influir mediante una posición participativa en conglomerado. Sobre la figura 2: *Existen diversas maneras de conectar con la realidad e interactuar, pero para efectos del tema consideramos los SGMA, más no es excluyente. $* *$ actuar pensando en el futuro es la inteligencia del plan, por lo que diseño y ejecución son críticos.

\section{TEMPORADA DE EJEMPLO}

Existen diversos ejemplos de cómo una política estatal o unas organizaciones comerciales en conjunto logran influir en el entorno. Siguiendo o no una base similar, pero si una intuición de por qué, entre ellas, destacamos las tendencias de los autos eléctricos en exposiciones comerciales de gran afluencia en la Unión Europea y Estados Unidos; la recolección de aguas lluvias en países con población en el margen de pobreza como Ruanda, Uganda o algunas favelas de Brasil; ingeniería microbiológica para combatir catástrofes ambientales, en diferentes países desarrollados. Procesamiento de residuos a base de celulosa para propósitos internos, entre otros. Desde luego, estos ejemplos son escasos para la cantidad de opciones que existen, pero destacamos estos por estar presentes en luga- res distantes del globo terráqueo, y merecen un nombramiento, aunque sea breve, porque ejemplifican como mediante ciertos sistemas se realizan proezas, se cambia el entorno y se independiza la organización de la volatilidad del mercado.

La comunidad autónoma de Madrid, siguiendo el reglamento (CEE) 1836/1983 conforme a como en 1993 la Unión Europea se pronuncia en consecuencia del tratado firmado por la misma el 7 de febrero de 1992, en Maastricht, en promoción de la creación de un crecimiento sostenible promulga posibilitar la adherencia voluntaria a sistemas comunitarios de gestión y auditorias medioambientales, nombrado como Ecomanagement and Audit scheme, en principio se promulgó para sistemas industriales pero la necesidad de aumentar la capacidad de impacto, la plataforma se dispuso para todo tipo de organización; ahora bien, puede ser certificada y ser una manera visible de conglomeración de organizaciones; desde otra perspectiva, en pro de los SGMA se requiere una ambición mayor que el crecimiento sostenible, pues en el momento actual existe la capacidad de realizar muchas de las fantasías que no eran posibles hace 22 años, muchas otras posibilidades han surgido y la investigación de procesos ha resultado en mayor eficacia para distintos propósitos, esta manera (EMAS) de agrupación macroorganizacional es un paso inicial al reconocimiento de la necesidad de cambio de mentalidad, pero la adherencia voluntaria en este caso no garantiza una participación activa, pero sí un reconocimiento de la necesidad como requerimiento mínimo de inclusión a un conglomerado oficial, obviamente adjuntarse tiene un costo de restructuración y capacitación, por lo que una organización basada en el benchmarking tiene grandes desventajas por la implementación de modelos genéricos; por lo tanto, en la fase de diseño y planteamiento se requiere atención para una implementación corta y con funcionamiento duradero y óptimo.

Alemania y su modelo energético fotovoltaico, en el momento es uno de los mayores ejemplos de posición participativa de conglomerados sociales, antes del desastre nuclear de Fukushima, Alemania se planteaba diversificar el origen 
de su suministro de energía dependiente de la combustión de sustancias fósiles e implementaba diversas investigaciones en modelos aplicables que proporcionaran una cadena de abastecimiento óptima, lo sucedido después de aquel desastre provocado por un tsunami movió las influencias políticas del país y se determinó que para el 2020 Alemania debería tener un suministro energético fotovoltaico cercano al $10 \%$ de la producción nacional anual de energía (I Corada, 2012), se implementaron para ello la fijación en principio del precio del kilowatt, distribución de subsidios para los interesados, y después de un tiempo la interconexión al cableado común para poder pasar los sobrantes de energía a la red eléctrica y ser vendidos, algunas empresas eléctricas cooperaron, otras si acaso ayudaron pero aún así Alemania alcanzó la meta faltando siete años para el horizonte establecido y ahora pretende ir por más. Dicho sistema planteó a partir de los sobrantes potenciar los beneficios del autoconsumo de energía, junto a un cambio de consumidor a productor energético en el ciudadano, este cambio propició que se creara toda una dinámica de mercado y un auge en la comercialización de paneles y empleo alrededor de la energía solar, reactivando la economía y fragmentado el mercado internacional de manera importante, al incentivar a los fabricantes nacionales a producir mayores cantidades y abaratar costes, según una economía de producción a escala, por supuesto, la abundancia de energía en el sistema se traduce en desperdicios y reducción del precio del kilowatt, pero debido al intervencionismo se logró subir el precio e incentivar a las personas del común aún más a participar de un mercado estable, y garantizando por algunos lustros prosperidad a pesar de que la duración de muchos paneles están alrededor de dos décadas. Debido a su popularidad, Alemania se convirtió en un país con mayor apego a este tipo energético, supliendo en gran medida termoeléctricas y reactores nucleares existentes, aún así la abundancia de energía y su baja eficiencia de reserva requiere de baterías que está superando a la oferta en el mercado inflando los precios de la industria, pero creando de nuevo un fragmento de negocio propicio para empresas especializadas en el almacenamiento eléctrico que, por cierto, requiere aún una carrera tecnológica destacable; antes de implementar dicho modelo de energía nacional se especulaba que arruinaría la economía alemana por su naturaleza de plan piloto y de subsidios que se debería distribuir para encaminar al proyecto. Uno de los cuestionamientos se refería a que mientras el fluido eléctrico tradicional funcione no hay garantía a largo plazo para el funcionamiento del sistema como conjunto, pero debido al entusiasmo y compromiso alemán el proyecto se convirtió en una hidra de mil cabezas que le permitió suplir los fallos de entes singulares, con la presión del conjunto que seguía suministrando fluido eléctrico, era imposible atacar el corazón del sistema si tenía millares de puntos de acceso que lo alimentan, por lo que el reto alemán debió estar no solo enfocado a hacer un cambio nacional, sino una economía alrededor del nuevo estilo de vida, donde el estado suministra ayuda a los canales de distribución, clave para el funcionamiento mientras el resto de ciudadanos contribuía con la producción, aquello generaría nuevos modelos de negocio y presión cultural para un progreso no atado directamente del suministro petrolífero. Básicamente se provocó el ambiente propicio para un cambio de mentalidad con formas de percibir la dependencia energética y económica del petróleo que es manipulado por terceros, que poseen grandes volúmenes; en resumen reduciendo la volatilidad de la estructura de costos y posibilitando una independencia respecto del fluido eléctrico se logró propiciar un cambio marcado en la Alemania de principios del siglo XXI, todo desde una apuesta por el conjunto de entes en la transformación del porvenir.

\section{CATARATAS}

La negación a una posición participativa del cambio genera una actitud conservadora y reacia en pro de sobrevivir en un ambiente competitivo, exigiendo especialización del ente para obtener rentabilidad. Ahora, bien comprendiendo como se encauza la realidad nihilista en las organizaciones, a nivel colombiano la directriz intervencionista, según el Plan Nacional de Desarrollo y el impulso nacional basado en la inclusión del concepto de competitividad en diversos sectores, pronto se incluyó una sectorización por interés asociado, siguiendo modelos extranjeros de crecimiento, basándose en deuda 
e inversión a gran escala para dotar al país de infraestructura, que posibilite a las redes comerciales un mayor dinamismo, aun así no logra repercutir en la sociedad de manera directa, pero si se apremia fuertemente la rentabilidad y efectividad empresarial basada en indicadores cuantitativos por sobre todas las cosas en el corto plazo, manteniendo la idea: "entre mejor es la acumulación de capital mayor noción de progreso es percibirle"; aclarando que lo anterior no del todo está mal pero tampoco es óptimo copiar modelos basados en ideales genéricos, el estado no logra promulgar una unidad nacional más allá de los intereses sociales, pronunciándose ocasionalmente sobre bienestar integral de las criaturas, por lo que se propicia un desarrollo industrial que apenas mitiga los impactos ambientales por fuerza de ley, sin lograr concebir la magnitud de sus problemas debido a la corrupción y violencia intranacional, corresponde, pues, a otros empezar a participar en el cambio y prever por el presente mientras el gobierno se da cuenta que mantener en ignorancia su población junto a la falta de un modelo de gestión acorde a las particularidades de la situación no es permisible, donde por la diversidad colombiana existen muchas potencialidades pero la superespecialización aun no funciona con la naturaleza del conjunto actual.

Da qué pensar el modelo alemán de energía, de un país ubicado en el hemisferio norte con una eficiencia de energía solar mayor a la de Colombia, que es un país del trópico, la excusa estará basada en que no poseemos la industria para la fabricación de paneles a escala, o que las importaciones no benefician a la economía colombiana en el corto plazo, pero Colombia posee una de las poblaciones de investigadores excepcionales reconocidas internacionalmente por su determinación, además de grandes capitales circulantes dispuestos a inversiones en capitales de riesgo, aun así en Colombia el poder del dinero supera al de las ideas, y entre mejor se dé la acumulación de capital mayor noción de progreso se percibe, por lo que la progresiva importación puede provocar la caída de los negocios similares locales, pero las re-copias de aquellos productos y un debido incentivo a la industria nacional generaría la cadena necesaria para la unión, claro está que es un supuesto, dentro de la lógica intervencionista alejada del neoliberalismo con tratados de libre comercio que exigen iguales condiciones de participación en el mercado, si Colombia no logra diversificarse en cambios progresivos en diferentes frentes del entorno, mediante la unión de los entes interesados, no se logrará el tan anhelado desarrollo promulgado por décadas, por lo que la posición pasiva seguirá siendo una incentivadora de la realidad nihilista, especialmente frente a las utopías ambientales de las organizaciones.

Por lo tanto, es imperativo conformar un conglomerado empresarial colombiano destinado a cambiar el destino de la nación con el ideal de bienestar integral y proyección futura, aplicando las buenas prácticas de diseño y planeación de proyectos y procesos internos de cada una de ellas para una eficaz y corta ejecución, provocando por sinergia el cambio de rumbo, pero ante todo, que prime la armonía entre entes relacionados.

\section{CONCLUSIONES}

Mediante la determinación de alcance, tiempo, costo, relacionados a los diferentes actores involucrados (voluntad-poder) y proyectos organizacionales (SGMA) se considera importante abordar la poción empresarial en relación a la comunidad circundante, en este caso, la situación colombiana es determinante en el cómo se plantea el escenario de la organización, lo que permitirá recolectar información y adaptarla a la consideración organización-sociedad-ambiente que se le hacen a los proyectos perseguidos, siendo imperioso entender cómo se pretende la cadena de influencia desde la organización hacia afuera y qué impacto registra a partir de lo planeado.

Respecto a los actores, evadir las visiones a largo plazo, en especial las utópicas, conlleva a ser casuísticos y operar en el acto, nublando la preparación hacia el cambio, confiando en documentos de planificación con limitación al cumplimiento, por lo que dicha pasividad hacia el largo plazo conlleva al estancamiento del pensamiento crítico, dispuesto de esta manera los autómatas están más cómodos dentro de lo 
establecido y no tienen por qué cuestionar lo que sucede alrededor, mientras estén satisfechos, debido a que los actores determinan gran parte del cursos de las cosas, los sistemas de gestión medioambiental deben evitar ser contagiados por la pasividad.

Comprendiendo la necesidad que se tiene en el ahora por el futuro, se comprende que la existencia de los agentes del cambio es fundamental, por lo que estos deberían estar más presentes en los procesos ejecutorios, la actitud y el clima que genere alrededor de sí permitirá caracterizar la dinámica de lo realizado, por lo que se requiere, que cada uno de los actores, a su manera, de forma armónica, se integren y actúen en pro del cambio sin entrar en discusiones ni líos que terminen siendo personales, pues debido a la necesidad de integración de las áreas contable, ingenieril y administrativa puede que se generen rencillas en el cómo se determinan los procederes próximos, los agentes del cambio son importantes y más aún si combinan un grupo de trabajo adecuado y comprometido con la causa; la contabilidad puede armonizar desde la perspectiva controladora y de realidad social a partir del poder de legitimar modelos contribuyentes a transformar la visión con una información más clara y concisa para el común de la gente dentro de la organización, sobre el área ingenieril resta decir mucho más, sin embargo, se requiere de un estudio a fondo de los problemas en la gestión y gerencia de proyectos de los mismos, por lo que en este documento solo se crea una noción de cómo su influencia práctica y utilitaria contribuye, o reduce la viabilidad de muchas de las ambiciones en el largo plazo.

En relación a las necesidades adquiridas definidas por McClelland (1987), se consideran a estas como influencias en el juicio de las personas en sentido de los SGMA para la organización y el entorno, siendo las necesidades de esta consideración y su relación directa con la interacción social provocadoras que determinan "logro, afiliación, poder" llenando de vicios la gestión ambiental, destacando en últimas que una constitución desde una perspectiva sistémica no puede ser determinada por influencias principales dispares a su naturaleza, dicho de esta manera, no puede verse alejada de la no- ción de bienestar integral para todas las criaturas del sistema, pues no se puede llevar a cabo algo para todos si está determinado por influencias individuales.

El nihilismo organizacional entorno a los sistemas de gestión medio ambiental hace parte de una derivación de la noción sobre la realidad como resultado de una desilusión y posterior resignación en la ignorancia de los fenómenos, por lo que coarta los planes a futuro y las actitudes de los actores, por supuesto el humano no puede vivir sin una motivación para sus procederes, lo que conllevan de esta manera a colocar los intereses por encima del propósito de los elementos existentes, por lo que, lo que importa para los sujetos no trasciende más allá del presente, destacándose el hedonismo y la rentabilidad en el ámbito comercial.

Para sobrellevar un SGMA que trascienda en el tiempo la integración contable e ingenieril es de suma importancia, deben entender los usuarios presentes y futuros que para constituir un producto que perdure, relativamente debe estar acorde al escenario y a lo existente, donde su mantenimiento permita la sostenibilidad del proyecto en el tiempo; tanto así que la calidad y el control de información sean manejados por parte de los gestores, todo el tiempo, mediante los sistemas de información y el flujo de recursos que registre la organización en el proyecto; adicionalmente se requiere la fuerza de un conglomerado de entes que promueva la armonía y coordine esfuerzos más allá de los intereses individuales, permitiendo cambiar la relación empresas-ambiente, creando una realidad distinta a la esperada y de esta manera perseguir la situación futura utópica.

Los sistemas de gestión medioambiental con una posición participativa en la modificación de escenarios organizacionales son una herramienta potencial para la disminución de la volatilidad del entorno, pues crea una posición de conexión recíproca entre el ambiente en que se enmarca la organización y la estructura que la constituye ,siendo posible considerarla como una conexión adicional a las normales en las visiones sistémicas, debido a que se genera alrededor un sentir de cambio y participación, se 
crea una dinámica de trabajo distinta que la gerencia deberá llevar a buen punto.

La motivación de las buenas utopías, en especial, las ambientales, ha creado diferentes puntos de vista en la época de la internet, pero en su mayoría son respetables según el caso, satisfacer las expectativas de un maravilloso sueño, tanto así que activa el precursor del movimiento mediante ansiedad y poder, pero lo esencial de una utopía es que genera un objetivo/horizonte a largo plazo, y como horizonte está comprendido como situación de integración de datos, logros aislados, momentos, actores, etc., por lo que se vuelve un futuro construible en cada decisión tomada en el presente, tanto así que se crea una incertidumbre en el abordar la imagen final y el sueño, al ser alcanzado no pude ser irreductible ni analizado solo desde la parte funcionalista de la gestión, como resultado se obtiene otro proceder pujante de la organización en pro de la armonía ambiente/estructura; es de aclarar que una organización que mueva sus SGMA por visiones utópicas debe estar razonablemente lucida para comprender la relación entre voluntad y poder, para no alejarse inmediatamente del objetivo original de constitución, por ir tras algo que se alcanza a largo plazo y con detalles.

\section{REFERENCIAS BIBLIOGRÁFICAS}

D. McClelland (1987). Citado por Eduardo Amorós, 2007 en: Comportamiento organizacional: en búsqueda del desarrollo de ventajas competitivas, adjunto a la Universidad Católica Santo Toribio de Mogrovejo, Peru, Facultad de Economía. Título seminario: Human Motivation (D. McClelland, 1987). Recuperado: www.eumed.net/libros-gratis/2007a/231/231. zip 15 abril de 2015 a las 6:00pm GMT5.

F. QUINCHE (2008) Una evaluación crítica de la contabilidad ambiental empresarial* rev.fac.cienc.econ., Vol. XVI (1), Junio 2008, 197-216, Universidad Nacional de Colombia. Recuperado de la dirección web: www.scielo.org.co/pdf/rfce/ v16n1/v16n1a14.pdf a las 9:19 p. m. lunes, 10 de abril de 2015 (GMT-5)

I. CORADA, (2012), El mercado de la energía solar fotovoltaica en Alemania, Oficina Económica y Comercial de la Embajada de España en Berlín; ICEX Instituto Español de Comercio Exterior. Otros documentos; recuperado de: www.aprean. com/internacional/estudios/FOTOVOLTAICA_ALEMANIA_2012.pdf a las 8:25 p. miércoles 8 de abril de 2015 (GMT-5)

J. SANTOS (2011), Palabras del presidente Juan Manuel Santos en la apertura del foro Expogestión Caribe 201, en sistema informativo del gobierno, Recuperado a las 7:19 p. m. lunes, 20 de abril de 2015 (GMT-5)de la dirección web: wsp.presidencia.gov.co/Prensa/2011/Abril/Paginas/20110428_06.aspx (GMT-5).

Kepner \& Tregoe (1989), El nuevo directivo racional, editorial McGraw Hill octubre 1993.

L. GONZÁLEZ, C. (1999). ¿Es la contabilidad medioambiental un paso hacia la sostenibilidad o un escudo contra el cambio?: El caso del sector eléctrico español.

Revista española de financiación y contabilidad.

L. Perea (2011), RSE, La responsabilidad social de la empresa en América Latina, capítulo 13, página 270, Banco Interamericano de Desarrollo, mayo 2011, recuperado de: http://idbdocs.iadb.org/wsdocs/getdocument.aspx?docnum=36193698, a las 7:30 pm el jueves 25 de junio de 2015 (GMT-5)..

O. AKTOUF (2015), 25 de febrero 6:00pm, referencia periodística recuperada: www.agenciadenoticias.unal.edu.co/ndetalle/article/planteamiento-administrativo-de-hace-200-anos-debe-actualizarse.html, recuperado el 15 abril de 2015 a las 6:00pm (GMT-5).

OIT, (1980) La consultoría de empresas: Guía para la profesión, publicada con la dirección de Milan Kurb; Oficina Internacional del Trabajo de Ginebra, Segunda Edición, Editorial LIMUSA, Noriega editores, 1980. 
P. COLLERETTE, La planificación del Cambio: Estrategias de adaptación para las organizaciones, Pelisle Editorial Trillas, título original: le Changement planifie, 1998.

REGLAMENTO (CEE) 1836/93 DEL CONSEJO, (1993) de 29 de Junio, por el que se permite que las empresas del sector industrial se adhieran con carácter voluntario a un sistema comunitario de gestión y auditoría medioambientales doce 168/1, de 10-07-93 p. 0001 - 0018; recuperado el lunes 10 abril de: www.aqa.es/doc/REGLAMENTO_CEE_183_93.pdf a las 9:00 pm de 2015 (GMT-5)

Reglamento EMAS, Guía práctica, manual de aplicación Recuperado de: eicat.cat/docs/docManualaplicacioReglamentEMAS..pdf a las a las 7:25 p.m. miércoles 1 de abril de 2015 (GMT-5)

SUNDER, Shyam (2005), Accounting theory and control. Editor Universidad Nacional de Colombia 